Eurostudia

\title{
Se sentir napolitain
}

Voir, vivre, écrire la ville avec Goethe, Sartre, Malaparte et Saviano

\section{Tobias Haberkorn}

Volume 8, numéro 1-2, 2012-2013

Paysages culturels de la modernité Kulturlandschaften der Moderne

Cultural Landscapes of Modernity

URI : https://id.erudit.org/iderudit/1026636ar

DOI : https://doi.org/10.7202/1026636ar

Aller au sommaire du numéro

Éditeur(s)

Le Centre canadien d'études allemandes et européennes

ISSN

1718-8946 (numérique)

Découvrir la revue

\section{Citer cet article}

Haberkorn, T. (2012). Se sentir napolitain : voir, vivre, écrire la ville avec Goethe, Sartre, Malaparte et Saviano. Eurostudia, 8(1-2), 149-169.

https://doi.org/10.7202/1026636ar

Tous droits réservés () Le Centre canadien d'études allemandes et européennes, 2014
STIUDIA 
Tobias Haberkorn

École des hautes études en sciences sociales / Freie Universität Berlin

$\mathrm{E}$ n voyage, Jean-Paul Sartre écrit que les napolitains sont " peut-être les seuls gens d'Europe dont un étranger peut dire quelque chose, même s'il ne passe que huit jours dans leur ville, parce que ce sont les seuls qu'on voie vivre de bout en bout $»^{1}$.

Ce que Sartre a pu ainsi dire des Napolitains se trouve réuni dans trois textes: la lettre "À Olga» de 1936 citée au-dessus, une nouvelle intitulée "Dépaysement» qu'il acheva en 1937 sans la publier ${ }^{2}$, et le reportage "Nourritures» de 19383. L'évolution entre la lettre et le reportage est considérable. Sartre raconte, puis fantasme sur Naples. En ce qui concerne l'importance de la vue, il va presque jusqu'à inverser son propos initial : qu'on les voie vivre de bout en bout, il semble vouloir dire, on ne peut pourtant pas (encore) dire grand-chose des napolitains. Si la ville est spectaculaire au sens strict $\mathrm{du}$ terme, il est frustrant de seulement la voir. L'impulsion napolitaine toujours trompeuse car insatiable in extremis - c'est qu'on vive la ville plutôt que de la voir vivre.

Ce déplacement du désir chez Sartre relève d'une expérience que nombre de visiteurs de Naples ont pu faire depuis des siècles et continuent à faire aujourd'hui ${ }^{4}$. Dans cet article, je présenterai une poignée de textes qui

\footnotetext{
${ }^{1}$ Jean-Paul Sartre, « À Olga » [été 1936] dans Lettres au Castor et à quelques autres. 1926-1939, éditées et annotées par Simone de Beauvoir (Paris : Gallimard,1983), 65. Souligné par Sartre lui-même.

2 Édition posthume dans CEuvres romanesques, éd. Michel Contat et Michel Rybalka (Paris : Gallimard, «Bibliothèque de la Pléiade », 1982), 1537-1557. L'idée de faire figurer cette nouvelle en tête du recueil Le Mur [1939] fut avortée par Sartre lui-même qui l'aurait jugée, en définitive, « ratée » (Voire le commentaire de Michel Rybalka, ibidem, 2127).

${ }^{3}$ Paru pour la première fois dans la revue Verve en novembre 1938, il est édité dans CEuvres romanesques, 2127-2129.

${ }^{4}$ La meilleure vue d'ensemble sur les témoignages littéraires sur Naples se trouve dans :

Raffaele Giglio (dir.), Napoli, città d'autore, 2 vol. (Naples : Edizioni Cento Autori, 2010).
} 
témoigne de la mise en scène littéraire de cette expérience, une mise en scène qui évolue de la réserve (sereine ou malheureuse) de celui qui regarde à la frénésie (enthousiaste ou maladive) de celui qui participe ou veut participer. Mon choix dépeint cette évolution comme littéraire et historique, mais elle peut être vécue individuellement par quiconque ayant le désir de Naples. Elle inclut touriste et autochtone, voyeurisme et vitalisme, sophistication et brutalité, art baroque et économie camorriste. L'itinéraire serait le suivant: Goethe, à la fin du $18^{\text {ème }}$ siècle, se contente de voir la ville ; Sartre, dans les années 1930, s'énerve contre le manque qui consiste à voir sans agir ; Malaparte, contemporain de Sartre, éveille les cinq sens pour sentir la ville ; et Roberto Saviano, de nos jours, mise le corps entier, en risquant son intégrité et sa sécurité propres, pour vivre la ville et ses contradictions les plus extrêmes.

Si ces exemples littéraires feront un récit de Naples, ils auront une portée plus générale pour au moins deux raisons: primo, parce qu'une relation littéraire à une ville n'est jamais que littéraire, elle est sensible, sensuelle et intellectuelle à la fois, c'est-à-dire une relation tout court. Un texte n'enregistre pas le son, l'aspect, l'odeur, la saveur ou le toucher d'une ville, il les représente. Et ses moyens à ces fins sont aussi riches qu'il comporte de métaphores. Secundo, parce que le choix littéraire révèle, dans son ensemble, une évolution des manières d'accéder non seulement à l'espace urbain, culturel et économique de Naples, mais à ces types d'espace en général. Ce « récit de Naples » parlera donc d'une ville sensuelle à travers sa littérature, mais j'espère qu'il dira des choses sur le rapport représentationnel et sensuel que les humains et leur culture entretiennent avec leurs habitats depuis plus de deux siècles.

\section{Voir NAPLES (AVEC ET CONTRE GOETHE)}

Das müssen Sie mir verzeihen, hatte ich zu Gambetti gesagt, daß Neapel für mich die allertiefste Provinz ist, ich kann es als nichts anderes bezeichnen, der Blick auf den Vesuv ist für mich eine Katastrophe, weil ihn schon so viele Millionen, möglicherweise Milliarden geworfen haben. (Thomas Bernhard, Auslöschung)

Le désir de voir Naples a été érigé en proverbe, à l'échelle européenne, par Goethe. Ainsi écrit-il dans son Voyage en Italie : "Von der Lage der Stadt und ihren Herrlichkeiten, die so oft beschrieben und belobt sind, kein Wort. "Vedi Napoli e poi 
muori!" sagen sie hier. "Siehe Neapel und stirb!" »5. La traduction de l'italien ignore le jeu de mots qui consiste dans l'homophonie de «muori » - « meurs » - et «Mori » - un village à la périphérie de Naples. Qu'importe, l'imaginaire européen a reçu la ville sous le Vésuve comme une sorte de chose ultime à voir. Goethe insiste sur le fait qu'il faut l'avoir vue de ses propres yeux : «[...] heute ward geschwelgt und die Zeit mit Anschauung der herrlichsten Gegenstände zugebracht. Man sage, erzähle, male, was man will, hier ist mehr als alles. »6

L'émerveillement durable de l'auteur se fonde dans la richesse de l'Anschauung, concept que je voudrais rendre par «vision » plutôt que par la traditionnelle « intuition » car c'est l'aspect concret et immédiat des choses qui est intenté plutôt que la capacité de l'esprit de se les représenter avec ou sans stimulus sensoriel. Une représentation verbale ou picturale de la chose vue n'est qu'un faible substitut : "hier ist mehr als alles », il faut être à Naples pour le voir vraiment.

L'épistémologie et l'esthétique de Goethe étaient celles du Augenmensch, « homme de l'œil » ${ }^{7}$. Il est frappant à quel point le poète semble convaincu que le seul sens de la vue lui permettra d'accéder à la connaissance d'un paysage et $\mathrm{d}^{\prime}$ une culture étrangers. Lorsqu'il tente de se familiariser avec l'économie du peuple napolitain, il s'en tient essentiellement à l'observation, ordonnant la confusion («ungeheures Gewirr») des personnages dans la rue selon leur apparence («Gestalt, Kleidung, Betragen, Beschäftigung») et jugeant cette opération plus aisée qu'ailleurs à cause de leur simplicité («weil der Mensch sich hier mehr selbst gelassen ist und sich seinem Stande auch äußerlich gemäß bezeigt $»^{8}$ ). Aucun dialogue avec un autochtone n'est rapporté (exception faite de ses fréquentations nobles).

Lorsque Goethe s'intéresse à la cuisine locale, il entreprend une énumération détaillée des aliments offerts sur les marchés ; il décrit leur aspect et surtout leur présentation qu'il juge partout plaisante à l'œil, mais il semble

\footnotetext{
${ }^{5}$ «Quant à l'entourage de la ville et ses splendeurs, qui ont été décrits et loués tant de fois, pas un mot. "Vedi Napoli e poi muori!" disent-ils ici. "Vois Naples et meurs !" », Johann Wolfgang von Goethe, Italienische Reise [1816-1817] (Munich : DTV, 1997), chap. 31, 2 mars 1787 ; traduction de T.H.

${ }^{6}$ « Aujourd'hui nous rêvâmes et passâmes notre temps à regarder les objets les plus splendides. On peut dire, raconter ou dessiner ce que l'on veut ; ici, tout est plus riche. », Goethe, Italienische Reise, chap. 31, 27 février 1787 ; traduction de T.H.

${ }^{7}$ La Farbenlehre en dit long. Voir aussi le numéro spécial «Augenmensch : Zur Bedeutung des Sehens im Werk Goethes » de la Deutsche Vierteljahrsschrift für Literaturwissenschaft und Geistesgeschichte (Nr. 75, 2001), édité par Dorothea von Mücke et David Wellbery.

${ }^{8}$ Goethe, Italienische Reise, chap. 55, 28 mai 1787.
} 
n'avoir rien goûté ni respiré les odeurs : du moins ne s'aventure-t-il à aucune description gustative. La supériorité épistémique de la vision est affirmée le plus explicitement dans les passages consacrés au Vésuve. Le spectacle du volcan en éruption déclenche l'éloge final de la synthèse visuelle : "Dies alles konnte von diesem Standpunkt das Auge mit einmal fassen, und wenn es auch die einzelnen Gegenstände zu mustern nicht imstande war, so verlor es doch niemals den Eindruck des großen Ganzen. »9

Le Naples de 1787 paraît donc, aux yeux du jeune Goethe ${ }^{10}$, comme un endroit d'une évidence aussi belle qu'instructive: la vision de l'art, de la nature et de la gaieté du peuple délectent l'œil et forment l'entendement. Une seule allusion aux choses que l'on ne peut pas voir : "Auf unserer Rückkehr [du Vésuve] nach Neapel wurden mir kleine Häuser merkwürdig, einstöckig, sonderbar gebaut, ohne Fenster, die Zimmer nur durch die auf die Straße gehende Türe erleuchtet. Von früher Tageszeit bis in die Nacht sitzen die Bewohner davor, da sie sich denn zuletzt in ihre Höhlen zurückziehen. ${ }^{11}$ Ces "grottes » où la population napolitaine se retire sont le décor d'un autre lieu commun napolitain, situé dans l'angle mort de l'Anschauung goethéenne. C'est l'image de Naples comme ville miséreuse, sale, voire abrutie. Que cela soit la cécité caractéristique du Augenmensch ou les bouleversements économiques et politiques ${ }^{12}$ qui engendreront à leur tour de nouvelles manières de voir dans les arts: les représentations du Naples de 1887, non plus capitale d'un royaume méditerranéen mais ville-réceptacle d'une région appauvrie, font paraître comme excessivement romantisants les clichés goethéens qui persistent pourtant dans la littérature de voyage du « Grand Tour».

\footnotetext{
9 «L'œil pouvait saisir tout cela d'un seul coup, et même s'il était incapable à considérer des objets distincts, il ne perdit jamais la vue d'ensemble. », Goethe, Italienische Reise, chap. 57, 2 juin 1787 ; traduction de T.H.

${ }^{10}$ Goethe publia ses mémoires de voyage trente ans après le retour d'Italie : s'est-il plus facilement rappelé des images que des odeurs ou saveurs ? La stratification sensuelle non seulement de la perception, mais aussi de la mémoire rentrerait en jeu. La philologie précise toutefois que la publication restait très fidèle aux journaux rédigés pendant le séjour de 1787/88. Cf. Herbert von Einem, « Nachwort», dans Johan Wolfgang von Goethe, Italienische Reise, vol. I (Munich : CH. Beck, 1981), 576.

11 «Au retour [du Vésuve], je remarquai des petites maisons sans étages, constructions étranges sans fenêtres où les chambres ne sont éclairées qu'à travers des portes qui donnent sur la rue. Du matin au soir les habitats s'assoient devant, jusqu'à ce qu'ils se retirent dans leurs grottes. », Goethe, Italienische Reise, chap. 32, 6 mars 1787 ; traduction de T.H. 12 Le risorgimento italien transforme Naples-capitale en Naples-ville-de-province, tandis que l'industrialisation européenne fait de la richesse agraire du Mezzogiorno une pauvreté infrastructurelle.
} 
Vers la fin du $19^{\text {ème }}$ siècle, ce sont des politiciens et journalistes italiens et non les étrangers en villégiature qui écrivent les textes les plus pertinents sur Naples. Ils mettent le doigt sur les conditions sanitaires et sociales souvent désastreuses. Les problèmes s'exacerbent dans les fondaci, anciens entrepôts transformés en résidences depuis l'explosion démographique du 17ème siècle. Voici la description d'un architecte, rapportée par l'historien Pasquale Villari dans une lettre de 1885 :

Ces fondaci [...] consistent généralement en une entrée inaccessible par la rue et en une petite cour, toutes deux extrêmement crasseuses et donnant sur un grand nombre d'habitations désastreuses, souvent en dessous des cabanes à chien, qui sont toutes, surtout celles du rez-dechaussée, privées d'air et de lumière et très humides. Dans celles-ci vivent, amoncelées, des milliers de personnes semblables, tellement avilies de la misère qu'elles ressemblent à des brutes plutôt qu'à des humains. Dans ces grottes, où l'on ne peut entrer à cause de la puanteur qu'émettent des poubelles amassées depuis des temps immémoriaux, on ne voit souvent qu'un tas de paille, destiné à faire mourir une famille entière, hommes et femmes confondus. Pour ne pas parler des latrines, car ce sont les rues voisines et les cours qui y suffissent ${ }^{13}$.

Les taudis sont décrits tantôt avec compassion et en urgeant vers l'action politique ${ }^{14}$, tantôt avec un dégoût presque haineux qui attribue à leurs occupants un caractère intrinsèquement vil et ignorant ${ }^{15}$. Les deux opinions ont en commun de souligner que le «vrai Naples» n'est pas dans son architecture belle quoique ruineuse, ni dans sa culture populaire truculente quoique archaïque, mais dans des lieux soustraits à la vue du voyageurvoyeur, dans lesquels il faut « descendre » comme dans des limbes dantesques. Le caractère physiquement éprouvant de telles descentes est souligné dans toutes les représentations du «ventre de Naples ». Il excède clairement le seul

\footnotetext{
${ }^{13}$ Pasquale Villari, Le lettere meridionale d'altri scritti sulla questione sociale in Italia, éd. par L. Marseglia (Turin : Bocca, 1885), 64-65. Cité d'après : Giglio, Napoli, città d'autore, vol. II, 138 ; traduction de T.H.

${ }^{14}$ Voir les écrits de Villari, Jessie White Mario, Axel Munthe et d'autres recueillis dans le chapitre « Verso los ventramento. Il racconto del degrado napoletano »dans Giglio, Napoli, citta d'autore, vol. II,131-161. Un assainissement au moins partiel de ces logements faisait l'objet de divers projets urbanistes depuis la fin du $19^{\text {ème }}$ siècle, de sorte qu'ils avaient disparu jusqu'aux années 1930.

${ }^{15}$ Voir les invectives du journaliste Yorick Figlio di Yorick (pseudonyme de Pietre Coccoluto Ferrigni) dans Giglio, Napoli, città d'autore, vol. II, 146.
} 
sens de la vue ${ }^{16}$ et ne saurait être plus contrasté avec les Anschauungen célébrées par Goethe. Vers la fin du 19 ème siècle, la littérature possède donc une idée assez développée de Naples comme ville de sensations extrêmes - beauté à voir, horreur à vivre - au point de former deux lieux communs opposés et/ou complémentaires ${ }^{17}$.

2. ÊtRe A NAPLes (SARTRE)

les odeurs, la crasse, et la mangeaille (Jean-Paul Sartre, « À Olga»)

Il est probable que Sartre n'ait lu aucun des textes cités précédemment. Toujours est-il que l'auteur de L'imaginaire confirme, au tout début de sa lettre « À Olga », les deux opinions schématiques sur Naples que je viens de mettre en relief. Son guide touristique vanterait le charme de la ville, alors que sa sœur l'aurait mis en garde que «Naples, [...] c'est des grandes maisons sales, mais la saleté ne suffit pas ${ }^{18}$. Ce que Sartre va dire de Naples s'articulera effectivement entre ces deux idées reçues, dont il - ou ses alter egos fictifs - n'auront cesse de vouloir se défaire. Après avouer, au moins par endroits, qu'il a été charmé19, Sartre se met à déprécier cette condition, s'efforçant de trouver sous la «crasse » napolitaine une épaisseur existentielle. Il désespère d'être à Naples, en italiques maximales : c'est la quête que les deux écrits subséquents à la lettre mettent en scène.

\footnotetext{
16 « Je visitai des souterrains semblables pour en arriver à un où, traversant le vacarme, ce fut difficile de vaincre la nausée qui m'assaillit par quatre des cinq sens, car seulement le goût n'y entrait pas. », Jessie White Mario, La miseria in Napoli (Napoli : Quarto Potere,1978 [1877]), 29-30, cité d'après : Giglio, Napoli, città d'autore, vol. II,150 ; traduction de T.H.

${ }^{17}$ En ce sens, c'est encore Goethe qui prononça le parfait poncif, quoique sans en avoir l'intention : lorsqu'il dit qu'à Naples l'« horrible » se joindrait au «beau » de façon à ce que les deux sensations « se neutralisent », il se référa à l'aspect infernal de la nature vésuvienne et non à celui des logements insalubres. "Das Schreckliche zum Schönen, das Schöne zum Schrecklichen, beides hebt einander auf und bringt eine gleichgültige Empfindung hervor. Gewiß wäre der Neapolitaner ein anderer Mensch, wenn er sich nicht zwischen Gott und Satan eingeklemmt fühlte. ", Goethe, Italienische Reise, chap. 36, 20 mars 1787.

18 Sartre, « À Olga », 63.

${ }^{19}$ Sa lettre ne cache pas un certain plaisir touristique : «Nous avons tout de suite été surpris et charmés », dit-il de sa première impression des ruelles napolitaines. Aussi les visites au café Gambrinus (très touristique) semblent lui avoir donné plus de satisfaction que ses descriptions ultérieures ne suggèrent. La vue sur la baie nocturne : " on ne peut rien rêver de plus luxueux », Sartre, « À Olga », 64, 71, 76.
} 
Puisqu'ils ont été rédigés successivement et au même propos, les trois écrits font la genèse d'une rencontre. Qu'en est-il de l'expérience napolitaine décrite au début, dans la lettre? En s'adressant à Olga, Sartre affirme pompeusement: «Je vais donc vous dire comment est Naples ${ }^{20}$. S'en suit un paragraphe sur la structure urbaine constituée de quelques belles rues neuves «traversées par des centaines de petites rues sordides » où l'on trouverait ce « Naples populeux » dont l'auteur relève le caractère hautement visible et à l'en croire si transparent. Mais voir ne suffit pas à Sartre. Ou ce qu'il voit lui donne à penser que voir ne suffit pas, à Naples.

Immédiatement après son éloge de la visibilité, Sartre se met donc à parler de choses qu'il ne voit pas : «Je suppose qu'ils se cachent à présent pour faire l'amour : à présent sous le règne de l'austérité fasciste ; mais il y a vingt ans, ils devaient le faire sur leurs seuils, ou bien sur leurs grands lits, toutes portes ouvertes. ${ }^{21}$ La tonalité ainsi établie, tonalité du voyeur qui cherche la transgression, dominera l'ensemble des trois écrits. On retiendra de la lettre une fixation sur la sexualité, la chair, la nourriture, la vie organique et animale $^{22}$; une grande importance attachée à l'odeur et à l'obscurité23 ; et sur un plan plus abstrait, le postulat d'une symbiose entre la vie de la rue publique, impudique, offerte au regard - et celle - cachée, entrevue, devinée des intérieurs. Les habitations au rez-de-chaussée (les bassi) paraissent à Sartre chargées de sens et de sensualité d'autant plus qu'elles se dérobent à son regard $^{24}$. La position du lit résume cette dialectique sensuelle :

\footnotetext{
${ }^{20}$ Sartre, « À Olga », 64.

${ }^{21}$ Ibidem, 65.

22 «Imaginez, jointe à ces misères, une extraordinaire liberté, sinon sexuelle, du moins charnelle : tous les enfants jusqu'à cinq ou six ans ont le derrière nu. [...] Et de fait, tout ce grouillement de derrières sales et de sexes, ça fait terriblement animal. Des animaux malades et souffreteux, une espèce qui grouille et qui est en train de crever, à la fois. » ; « D'une façon générale, les rues regorgent de nourritures : on vend des noix, des épis de maïs bouillis, des fèves grillées, des poissons, des crustacés, de petites pieuvres. », ibidem, 66 et 83 .

${ }^{23}$ « Toutes ces rues sont si étroites que les pièces qui s'ouvrent sur elles, malgré la violence du soleil, restent obscures en plein midi. Obscures mais non fraîches. Il doit y faire tiède, une de ces tiédeurs qui développent les odeurs des choses et des gens, car de chaque pièce sombre émane une bouffée d'odeur complexe et puissante, une odeur de sueur, de fruits, de friture, de fromage et de vin qui nous baignait au passage et dont nous ne sortions que pour nous enfoncer dans une autre odeur qui contenait, en proportion différente, les mêmes éléments. », ibidem, 78.

24 « Tout est dehors, comprenez-vous, mais tout reste attenant, soudé, organiquement relié au dedans, à la coquille : ce qui donne du sens à ce qui se passe dehors, c'est la caverne sombre qui est derrière et où la bête rentre dormir le soir, derrière d'épais volets de bois. », ibidem, 79 .
} 
Du même lit, on ne saurait dire s'il est dehors ou dedans. Sans doute, vers les onze heures, ils rentrent dans les odeurs lourdes de la chambre, ils tirent les volets et se mettent au lit, marinant dans cette atmosphère pleine de relents. Et cette fois, le contact avec le dehors est rompu, le corps a ravalé son estomac : du dehors on ne voit plus que des volets pleins, fermés par des barres de fer, on croirait qu'il n'y a là que des échoppes abandonnées pour la nuit, on ne soupçonne pas toutes les respirations et toutes les digestions qui se poursuivent dans l'ombre. Mais, dès qu'une femme est malade et reste au lit dans la journée, ça se passe au grand jour et chacun peut la regarder. Nous avons vu, hier, en passant, une malade, très pâle, couchée dans le grand lit nuptial, la tête tournée vers la rue et qui regardait passer des gens de ses grands yeux fiévreux ${ }^{25}$.

Un souci que Sartre exprime déjà dans la lettre, c'est que toutes ces impressions ne feront qu'anecdote, qu'elles ne permettront pas de saisir « l'aspect général de ces rues », « le coup d'œil d'ensemble qu'elles offrent quand on entre dans l'une d'elles ${ }^{26}$. S'il semble encore compter, ici, sur les vertus d'une "vue d'ensemble», cette option sera clairement écartée par Audry, protagoniste de «Dépaysement». Celui-ci, raconté à la troisième personne,

aurait voulu tout voir, mais on en peut pas saisir à la fois tous les petits échanges protoplasmiques qui se font, avec une rapidité hésitante, dans une ruelle de Naples. [...] Il y avait quelque chose à prendre dans ce Naples misérable qu'il venait de quitter. Quelque chose dont on eût pu jouir, un sens - peut-être était-ce même le sens de Naples. Mais il n'avait pas su en profiter, c'était à chaque fois la même chose : ces ruelles le touchaient trop pour qu'il pût les voir comme elles étaient ${ }^{27}$.

La dépréciation de la vue dans «Dépaysement» ne pourrait être plus nette ${ }^{28}$. Il s'agit d'être à Naples, et non seulement pour la voir de ses propres yeux. Une des torsions du texte sartrien consiste dans le fait qu'il met une telle emphase

\footnotetext{
${ }^{25}$ Sartre, « À Olga », 80. On reverra la femme couchée dans les deux nouvelles.

${ }^{26}$ Ibidem, 85.

${ }^{27}$ Sartre, « Dépaysement », 1542.

${ }^{28}$ Ainsi lit-on sur la première page : «Des faits, des toutes petites faits : on les attrape quand ils passent. Audry se dit avec dégout : "des impressions de voyage." » ;ou encore : " Il avait pris des points de vue sur la ville; il avait vu Naples du Vésuve, Naples du Pausilippe, Naples du Palais-Royal. Et puis après ? Espérait-il un jour la reconstituer en mettant tous ses aspects bout à bout ? Ce n'est pas ainsi que les villes se dévoilent. Il suffit parfois de regarder un pavé déchaussé, de respirer une odeur, ça y est, la ville est là, autour de vous. », ibidem, 1537.
} 
sur les concepts de sens et être, qu'il est presque impossible de le prendre au sérieux. La mise en scène d'un touriste séparé de l'être napolitain par la balustrade d'une terrasse de café est on ne peut plus simple: «D'ailleurs il n'était pas à Naples : il voyait les Napolitains descendre et monter les étroits trottoirs de la via Roma, mais la barrière de bois qui entourait la brasserie Gambrinus le séparait d'eux plus sûrement que s'ils avaient évolué sur une scène. ${ }^{29}$ Combien doit-on être naïf pour croire que la barrière d'une brasserie est l'obstacle principal à une communauté ontologique avec un milieu étranger? Audry en tout cas se ravise, mais même une visite des ruelles sordides de Naples, où il voit pour l'essentiel ce que Sartre avait déjà raconté dans sa lettre ${ }^{30}$, ne le lui permet pas de se sentir napolitain. «Et pourtant Naples existe. Il suffirait d'un rien... c'est-à-dire qu'il me faudrait une aventure. Pas grand-chose, quelqu'un qui m'adresserait la parole - et alors je sentirais peut-être que je suis à Naples. » ${ }^{31}$

Cette aventure se produit lorsque Audry est interpellé dans la rue par un certain Rossi qui l'emmène boire du vin, rencontrer son ami Renato, visiter un bordel, et finir le vin dans le basso de Renato qui joue de la mandoline. Le triomphe final d'Audry est de bonne foi : quand il se rend compte que tous les autres touristes, les magistrats et même le secrétaire du parti fasciste dorment alors que lui vient de sortir d'un de ces intérieurs tièdes et mystérieux, son cœur «se mit à battre plus vite ; une ombre énorme s'était levée devant lui : "Cette fois ça y est, dit-il à voix haute : je suis à Naples" »32. Ce n'était donc pas le seuil d'un café, mais ceux d'un bordel et d'un basso qui le séparèrent de Naples.

Le fait que Sartre jugea cette nouvelle «ratée » porte à croire qu'il s'est rendu compte du pathos grossier, voire ridicule de ce dénouement. Pourtant, si son intention avait été de ridiculiser la naïveté d'un touriste, il aurait pu la publier. Notons que les deux expériences initiatiques d'Audry sont précisément celles que Sartre n'a pas vécues alors qu'il en fantasmait : si l'on se tient au témoignage de sa lettre, les intérieurs napolitains lui restèrent inaccessibles et il n'a pas assisté, ni en voyeur ni en acte, à une de ces scènes sexuelles qu'il soupçonnait partout. Il dit ne pas avoir vu de bordel à Naples,

\footnotetext{
${ }^{29}$ Sartre, « Dépaysement », 1538.

${ }^{30}$ Un exemple entre beaucoup : « Des chambres impudiques et secrètes : elles vous jettent au visage leur chaleur organique mais elles ne se livrent pas. ». Aussi voit-il la femme couchée : «Audry la regarda un instant avec un peu de concupiscence, puis, dégoûté de lui-même, il s'éloigna à grands pas. », ibidem, 1541, 1542.

${ }^{31}$ Ibidem, 1544.

${ }^{32}$ Ibidem, 1557.
} 
inférant que "[l]e fascisme y a mis bon ordre et la prostitution doit $\mathrm{s}^{\prime} \mathrm{y}$ cacher ${ }^{33}$. Sartre semble s'être servi de la fiction pour pénétrer dans des lieux qui dans la réalité lui étaient inconnus ou introuvables.

Dans «Nourritures», seul texte sur Naples effectivement publié par l'auteur, les conditions pour y être ont été atténuées. Le protagoniste, un narrateur homodiégétique également échoué au Gambrinus, part se balader dans les ruelles napolitaines où il découvre «la vérité de la nourriture », à savoir sa "parenté immonde» avec l'amour ${ }^{34}$. «Nourritures» est de loin le plus court des trois textes, et le plus réussi sur le plan poétique : les banalités touristiques de la lettre et la bonhomie excessive du personnage Audry ont été enlevées au profit d'une description linéaire, condensée et métaphoriquement riche de la manière dont les napolitaines «jouissaient avec une indolence sensuelle de leur vie organique $»^{35}$. La dramaturgie est simple et efficace : ici aussi le narrateur a droit à son " "Ça y est : je suis à Naples" » final, mais pour cela il ne lui faut pas d'événement extraordinaire : c'est une simple ballade dans les rues grouillantes qui est à même de déclencher en lui une altération mentale, physique et, si l'on veut, ontologique. Elle se réalise à travers un délire sur la chair, matière commune du manger et du baiser, qui est achevé par la vision de la femme couchée dans le basso:

$\mathrm{Au}$ fond d'un trou il y avait une forme dans un lit. C'était une jeune femme, une malade. Elle souffrait, elle tournait la tête vers la rue, sa gorge faisait une tâche tendre au-dessus des draps. Je marrêtai, je la regardai longtemps, j'aurai voulu promener mes mains sur son cou maigre... Je me secouai et m'éloignai à grands pas. Mais trop tard: j'étais pris. Je ne voyais plus que de la chair, des fleurs de chair misérables, qui flottaient dans une obscurité bleue, de la chair à palper, à sucer, à manger, de la chair mouillée, trempée de sueur, d'urine, de lait ${ }^{36}$.

Si l'on considère les trois textes de Sartre dans leur ensemble, la continuité des descriptions est frappante: toutes les images fortes de «Nourritures » et de «Dépaysement » sont déjà présentes, parfois jusque dans leur texture lexicale, dans «À Olga ». Le seul élément totalement absent de la lettre, l'aventure

\footnotetext{
33 Sartre, « À Olga », 87.

${ }^{34}$ Sartre, « Nourritures », 2127-2128.

${ }^{35}$ Ibidem, 2128. La formule apparaît déjà dans « Dépaysement » : « Tous ces gens semblaient tournés vers eux-mêmes ; ils ne rêvaient même pas : entourés de déchets vivants, à demi digérés, maculés, d'écailles, de trognons, de viandes obscènes, de fruits salis et ouverts, ils jouissaient avec indolence de leur vie organique », 1542.

${ }^{36}$ Ibidem, 2129.
} 
d'Audry, a été effacé dans le dernier texte. Une évolution doit pourtant être reconnue : il semblerait que Sartre ait vraiment trouvé le sens de Naples, ou plutôt son sens de Naples à lui, dans «la parenté immonde de l'amour et de la Nourriture ». C'est une interprétation originale et plausible, en tout cas présentée de manière très suggestive, de ce qui peut arriver à quelqu'un qui se plonge dans Naples sensuellement. Le mode d'accéder à ce sens n'est pas celui du voyeurisme encore dominant dans la lettre : ce que Sartre observe et décrit à Olga le laisse charmé, parfois perplexe, mais globalement insatisfait; il voudrait vivre la ville plutôt que de la voir. Mais ce n'est pas non plus le vitalisme empressé et naïf d'Audry qui réalise le « sens de Naples » : l'échec de «Dépaysement» est d'autant plus gênant qu'il passe par la prétendue conquête (purement fictionnelle du point de vue de Sartre) de deux haut-lieux du poncif napolitain, le bordel et le basso, où le pittoresque abonde (prostitution grotesque, bonté mélancolique et sournoise des autochtones, mandoline, odeur de tomates, canzone).

« Nourritures » nous fait comprendre que l'expérience la plus valable, la plus vraie pour ainsi dire, n'est pas dans le voir ni dans le vivre, cru ou inventé, d'une extériorité : elle est dans la dérive hallucinatoire d'une voix narrative. Sartre a mis l'aggravation métaphorique de ses perceptions premières au cœur de son texte final; c'est cela qu'il semble vouloir retenir de ses multiples tentatives d'être à Naples.

Ce dénouement porte à une certaine modestie. Il est impossible, pour un étranger qui n'y passe que quelques jours, de devenir napolitain. Pour être à Naples tout de même, au sens le plus complet possible, le mieux est de se concentrer sur soi-même et d'extérioriser les divagations de la propre conscience (ou mémoire) perceptive. Le fait est que Sartre a bel et bien été à Naples - en italiques ou non - et que les choses qu'il y a vues et vécues lui ont inspiré un certain nombre de descriptions et de réflexions qu'il exprime au premier chef, de façon déjà éloquente et assez copieuse (vingt-six pages), envers Olga. Dans ce sens, sa lettre est la représentation tout à fait respectable d'une expérience vécue. " Nourritures » va plus loin dans le même genre, sans prétendre qu'une quelconque aventure pourra transporter l'étranger sur un palier ontologiquement autre, l'arracher à sa condition de touriste. Cet arrachement n'aura lieu que dans la conscience du protagoniste, dans l'élaboration métaphorique des informations sensuelles et partant dans le langage. Le dépassement $\mathrm{du}$ voyeurisme et du vitalisme, conditions de l'étranger qui s'obstine à entrer dans un milieu inconnu, ne peut avoir lieu que dans le travail des métaphores, dans l'élaboration d'un style, dans une forme poétique. 
Par la suite j'aimerais montrer comment une telle forme poétique se dessine de façon plus assurée et efficace chez Curzio Malaparte et Roberto Saviano, l'un résident de Naples et l'autre autochtone, qui ont tous les deux écrit des romans sur la ville, l'un sur la libération de 1943, l'autre sur le régime contemporain de la camorra.

\section{SENTIR NAPLeS (MAlapARTE)}

Une odeur étrange flottait dans l'air. Ce n'était pas l'odeur qui descend, au coucher du soleil, des ruelles de Toledo, de la piazza delle Carrette, de Santa Teresella des Espagnols. Ce n'était pas l'odeur des friteries, des tavernes, des urinoirs, nichés dans les ruelles fétides et sombres, qui montent de la via Toledo vers San Martino. Ce n'était pas cette odeur jaune, opaque, gluante, faite de mille effluves, de mille troublantes exhalaisons, "de mille délicates puanteurs" comme disait Jack, que les fleurs fanées, amoncelées aux pieds de la Vierge dans les tabernacles aux coins des rues, répandent dans toute la ville à certaines heures du jour. Ce n'était pas l'odeur du sirocco, qui sent le fromage de chèvre et le poisson pourri. Ce n'était pas non plus cette odeur de viande cuite qui, vers le soir, monte des bordels et se répand à travers Naples, cette odeur dans laquelle Jean-Paul Sartre, marchant un jour dans la via Toledo, sombre comme une aisselle, pleine d'une ombre chaude vaguement obscène, respirait la parenté immonde de l'amour et de la nourriture. Non ce n'était pas cette odeur de chair cuite qui pèse sur Naples vers le coucher du soleil, quand la chair des femmes à l'air bouillie sous la crasse. C'était une odeur d'une pureté et d'une légèreté extraordinaire : maigre, légère, transparente, une odeur de mer poudreuse, de nuit salée, l'odeur d'une antique forêt d'arbres en papier ${ }^{37}$.

Dès l'ouverture de La pelle, un roman de 350 pages, Malaparte saisit la ville par son odeur. Il le fait par une série d'antiphrases qui est non seulement un catalogue de parfums napolitains mais aussi un catalogue des moyens dont dispose l'italien (et bien d'autres langues) pour représenter des odeurs. Contrairement aux autres sens, il n'existe pas (en italien pas plus qu'en français ou en allemand) un vocabulaire spécifique pour l'odorat. C'est-à-dire qu'il y a

\footnotetext{
${ }^{37}$ Curzio Malaparte, La peau [1949], trad. de l'italien par René Novella (Paris : Denoël, collection « folio », 1996), 23-24.
} 
bien des mots qui servent prioritairement à décrire la qualité du son, de l'aspect, du toucher ou de la saveur d'un objet, mais pour spécifier une odeur, il faut en général nommer un objet de référence ("ça sent les roses») ou recourir, par métaphore, à une autre catégorie sensuelle (« un parfum sucré »). Bien sûr, tous les attributs sensuels sont métaphoriques à un certain degré et par conséquent transposables les uns sur les autres, mais il se trouve que nos langues européennes sont particulièrement limitées - et par conséquent la métaphore est nécessaire - dans le domaine olfactif. Or, comme nous avons lu chez Sartre, Villari et d'autres, l'odeur est une des sensations les plus fortes et les plus dépaysantes dans les rues et les intérieurs napolitains. Mais aucun de ces auteurs n'en a fait le vecteur principal de l'expérience napolitaine.

Dans le passage cité, Malaparte arbore une multitude de manières pour objectiver l'odeur de Naples : elle a un temps et un lieu (au coucher du soleil, dans certaines ruelles, au pied des Vierges à certaines heures...); elle a des qualités optiques et tactiles («jaune, opaque, gluante»); elle est trois fois composite («faite de mille effluves, de mille troublantes exhalaisons, "de mille délicates puanteurs" »); elle provient de produits et d'endroits napolitains notoires (friteries, tavernes, urinoirs, fleurs fanées...) ; elle porte un nom propre («sirocco») qui ne reste pas sans explication («qui sent le fromage de chèvre et le poisson pourri »); elle est un objet de conversation («comme disait Jack»); et elle est - attention - un objet littéraire (« cette odeur dans laquelle Jean-Paul Sartre [...]»).

Le fait que Malaparte ouvre toutes ces perspectives pour évoquer la ou les odeur(s) de Naples témoigne déjà d'une sensibilité olfactive inhabituelle, et on pourrait dire que la prosodie parfaite de son paragraphe ajoute un rythme à l'odeur. Mais ce qui rend ce tableau olfactif tout à fait extraordinaire est la manœuvre rhétorique finale. Malaparte énumère des odeurs typiquement napolitaines seulement pour les récuser («ce n'était pas [...]») et pour leur opposer, en dernier lieu, un nouveau parfum, sa création propre pour ainsi dire. Non, ce soir-là, Naples ne sentait pas tout ce à quoi on pouvait s'attendre ou dont a pu lire, ni les fleurs, ni la friture, ni l'urine, ni même la «chair cuite » de Sartre. Ce soir-là, ce n'était aucun de ces arômes qui, pour le meilleur et le pire, correspondent au folklore - et donc à la réalité - napolitain. Ce soir-là, « [c]'était une odeur d'une pureté et d'une légèreté extraordinaire : maigre, légère, transparente, une odeur de mer poudreuse, de nuit salée, l'odeur d'une antique forêt d'arbres en papier. »

Cette ligne est efficace non seulement parce qu'elle renchérit sur les descriptions synesthésiques d'avant («maigre, légère, transparente » remplace 
«jaune, opaque, gluante»), mais surtout par l'image invraisemblable qui la conclut. Qu'est-ce qu'une "antique forêt d'arbres en papier»? Une chose au moins est sûre, c'est qu'elle a une odeur, celle que Malaparte vient de détailler. La qualité sensuelle de son écriture réside dans le fait qu'elle combine la virtuosité de la représentation métaphorique et/ou objectivante des cinq sens avec la liberté de l'imagination. Sartre avait cherché le sens de Naples dans une expérience exotique et il l'avait trouvé, péniblement, dans la description d'une hallucination. L'écriture malapartienne ne décrit pas une hallucination, elle est en quelque sorte une hallucination. Rien n'y marque une altération psychique: elle prétend que ce soir-là, Naples sentait objectivement «une antique forêt d'arbres en papier ».

Malaparte pose donc l'invention contre l'introspection: son style est expressionniste, excentré par rapport à la conscience perceptive et non concentré sur elle comme chez Sartre. La sensualité de son écriture (et, par le biais de la lecture, celle de Naples) en est sensiblement augmentée. Dans cette écriture, la falaise de Sorrente prend l'air d'« une dure gencive de marbre vert », tandis que le Vésuve ressemble «à un vieil os, décharné et poli par la pluie et le vent $»^{38}$. La mer a tantôt « la couleur du vin, qui est la couleur de la mer dans Homère ${ }^{39}$, tantôt celle, "verte et brune [,] de la peau de crapaud » ${ }^{40}$, si elle ne se présente "striée de courants, les uns blancs, les autres verts, d'autres pourpres » lui donnant "une sonorité argentée ${ }^{41}$. La ville est organique : au crépuscule, moment de prédilection de Malaparte, "les sons, les couleurs, les voix, et cette saveur de mer, cette odeur de laurier et de miel, qui sont la saveur et l'odeur de la lumière de Naples » viennent « $[u] n$ à un, comme les daims, les sangliers dans la forêt, [...] chercher refuge dans la tiédeur de la nuit » ${ }^{42}$. Un mur " tout lézardé par le temps et les saisons » paraît "quelque chose de vivant, un mur de chair où apparaissaient toutes les aventures de la chair humaine, depuis la rose innocente de l'enfance jusqu'à la verte et jaune mélancolie de l'âge déclinant ${ }^{43}$.

La liste des exemples pourrait continuer. Le narrateur croise les catégories sensorielles et il accorde à l'imagination une autonomie qui invente ou anime des objets de son expérience sensuelle, chose qui rend la représentation de la ville plus spectaculaire et plus riche que chez tous les autres auteurs. Dans

\footnotetext{
${ }^{38}$ Malaparte, La peau, 54.

${ }^{39} \mathrm{Ibidem}, 54$.

${ }^{40}$ Ibidem, 73.

${ }^{41}$ Ibidem, 318.

${ }^{42}$ Ibidem, 164.

${ }^{43}$ Ibidem, 163.
} 
« Dépaysement », Sartre aussi s'était laissé emporter par l'imagination vers des lieux qui lui restèrent empiriquement inconnus. Mais à la différence de Malaparte, il le faisait pour satisfaire ses idées reçues, ou plus précisément ses obsessions. Malaparte est au-delà de l'idée reçue, il la transgresse dès l'abord et avec ostentation.

En ceci, c'est dans la représentation sensuelle que son écriture devient supérieure et surtout plus originale que celle de Sartre. Mais est-elle aussi plus «vraie » ou plus « authentique»? Malaparte était conscient de l'embarras que son style était à même de provoquer, surtout à cause du contenu moral de ses livres qui est comme le contenu sensuel sincèrement invraisemblable. Il fait dire à un de ses personnages dans La pelle: "Qu'importe [...] si ce que Malaparte raconte est vrai ou faux. Ce qui importe c'est la façon dont il le raconte ${ }^{44}$. Son énorme allégorie de l'expérience sensuelle (et morale) du Naples des années 1943/44 est composé sous le paradigme de l'art, non celui de la vérité (qu'il ne revendique jamais, contrairement à Sartre). Dans cette ville, des choses incroyables se passent. Dans la mesure où Naples est hallucinant, il faut le présenter comme tel, avec les moyens poétiques adaptés. Malaparte ajoute à l'invraisemblable au lieu de le réduire, il renonce à la représentation réaliste de la ville et d'une conscience qui s'y promènerait. Aggraver l'invraisemblable, omettre la distinction du vrai et du faux, de l'authentique et du mis en scène, dans la mesure où celles-ci n'ont que peu de sens : voilà la réponse malapartienne au défi de représenter la ville. Nous allons voir que le prochain et dernier auteur choisi, Roberto Saviano, n'a pas pu choisir cette voie. Sa volonté de dire la vérité sur Naples le pousse à un empirisme charnel.

\section{NAPLES A CORPS PERDU (SAVIANO)}

Io so. Ma non ho le prove. (Pier Paolo Pasolini)

Contrairement à Goethe, Sartre et Malaparte, Roberto Saviano est né et a grandi à Naples. Ce qui paraît invraisemblable à d'autres l'est donc moins pour lui. Il n'est pas fasciné par la densité urbaine, sociale ou culturelle de la ville; son sujet est plus abstrait et de ce fait moins accessible à l'expérience sensuelle: c'est le règne économique et militaire de la camorra, la mafia napolitaine. Gomorra est un véritable roman d'investigation qui tient du

\footnotetext{
${ }^{44}$ Malaparte, La peau, 364. La traduction diffère du texte original, qui pose la question de l'art : «La questione da porsi è un'altra : se quel ch'egli fa è arte, o no. », Curzio Malaparte, La pelle (Milan : Adelphi Edizioni, 2010 [1949]), 284.
} 
reportage social, de l'enquête criminologique et de l'autobiographie, tout en s'appuyant sur un appareil de chiffres digne d'un traité en macroéconomie. Ce brassage d'éléments se veut aussi près de la vérité que possible. Saviano est un vériste; il prétend découvrir la réalité nue et immédiate des mécanismes secrets et officieux de la camorra. Toutefois, pour s'approcher le plus possible de cette réalité, il choisit des moyens littéraires. Les éléments poétiques dans ses textes sont précisément destinés à ancrer cette réalité dans le monde sensible.

Dès le début de Gomorra, Saviano déplore l'inadéquation entre le monde économique et l'intuition :

La première fois que j'ai vu un bateau chinois accoster au port, j'ai cru me trouver devant toute la production du monde. Mes yeux ne parvenaient pas à compter, à estimer le nombre de conteneurs déchargés. Je ne réussissais pas à faire le calcul. Ça peut sembler difficile à croire, pourtant je m'y perdais, les chiffres étaient trop grands, ils se mélangeaient dans mon esprit ${ }^{45}$.

L'économie paraît à la fois matérielle et irréelle, comme les objets qu'elle véhicule :

C'est une bizarrerie difficile à comprendre, mais les marchandises ont leur magie, elles peuvent être à un endroit sans y être, arriver sans jamais vraiment arriver, coûter cher au client tout en étant de qualité médiocre, et valoir peu aux yeux de la douane tout en étant précieuses $^{46}$.

Le but de Saviano va donc être de trouver un moyen pour rendre les structures économiques accessibles à l'intuition. En quelque sorte, ce qui l'occupe est la dialectique entre savoir et sentir: il n'est pas apaisé par la connaissance abstraite des phénomènes, il veut toucher, respirer et vivre de façon brutale tout ce qui l'inquiète. Il évoque la nécessité de "modifier l'échelle de son imagination " pour comprendre, par exemple, comment l'immense production textile chinoise $(70 \%$ des exportations) peut « s'appuyer sur une marche aussi petite que le port de Naples ${ }^{47}$. Le roman s'ouvre sur ce parfait décor, endroit où les flux de l'économie coagulent, où les marchandises deviennent visibles et palpables un instant avant de disparaître dans les camions et dans les entrepôts majoritairement gérés par la camorra.

\footnotetext{
${ }^{45}$ Roberto Saviano, Gomorra, trad. de l'italien par Vincent Raynaud (Paris : Gallimard, collection « folio », 2007), 21.

${ }^{46}$ Ibidem, 17.

${ }^{47}$ Ibidem, 19.
} 
Pour ancrer la fonction du port dans le sensible, Saviano mobilise un grand nombre de métaphores. Il le décrit comme une «blessure» ou encore comme un «trou noir» où "la structure moléculaire des choses semble se décomposer ». Les bateaux s'approchent de la darse « comme des petits attirés par les mamelles de leur mère ${ }^{48}$. L'image la plus crue mélange motifs bibliques, industriels et charnels : « le port joue le rôle du chas d'une aiguille et les bateaux celui du chameau qui passera au travers. Proues qui se heurtent, énormes bâtiments en file indienne qui attendent à l'extérieur du golfe de pouvoir entrer, chaos de poupes qui tanguent et émettent des plaintes métalliques: tôles et boulons qui pénètrent lentement dans le petit trou napolitain, un anus de mer qui s'élargit et martyrise les sphincters. ${ }^{49}$

Cet engouement pour les métaphores organiques et physiques témoigne déjà des efforts pour rapprocher abstraction et corps propre. Il va donner suite à des méthodes d'investigation tout aussi physiques : dans le premier chapitre, Saviano raconte comment il se fait engager par un commerçant chinois qui transforme de beaux immeubles bourgeois tout près du port dans des énormes stocks de marchandise. L'auteur participe à l'abattement des murs, aux déchargements en pleine mer, au transport et à l'interminable rangement des cartons. Les produits contrefaits se confondent avec les originaux, rendant les marchandises trafiquées

moins visibles que les cigarettes puisqu'elles ne seront pas distribuées par un circuit parallèle. Comme si elles n'avaient jamais été transportées, comme si elles poussaient dans les champs et que quelque main anonyme les avait récoltées. Car si l'argent n'a pas d'odeur, les marchandises, elles, en ont une. Pas celle des mers qu'elles ont traversées, pas celle des mains qui les ont produits ou de l'huile des machines qui les ont assemblées. Les marchandises ont leur propre odeur, qui naît sur l'étal du commerçant et qui ne disparaît qu'une fois chez le consommateur ${ }^{50}$.

La marchandise est devenue abstraite jusqu'à son odeur, qui est l'odeur caractéristique du carton désinfecté. Les efforts de Saviano pour surmonter cette abstraction frisent l'obsession. Dans les passages les plus saisissantes du roman, il se met lui-même en danger, il soumet son corps et son esprit aux rouages de production et de contrôle par lesquels la camorra gouverne Naples et la Campanie.

\footnotetext{
${ }^{48}$ Ibidem, 17-18

${ }^{49}$ Ibidem, 19.

${ }^{50}$ Ibidem, 35.
} 
Un épisode qui met en évidence la quête de sentir et non seulement savoir se trouve dans le chapitre "Béton armé» dédié aux casalesi, les clans originaires de Casal di Principe. Saviano dit avoir fait la connaissance physique de leur pouvoir :

Le pouvoir des clans était le pouvoir du béton. Sur les chantiers, je sentais physiquement en moi, dans mes tripes, toute leur puissance. Pendant plusieurs étés, j'avais travaillé sur des chantiers. [...] Un boulot infernal [pétrir le ciment] que je n'ai jamais su très bien faire, un métier qui peut rapporter pas mal d'argent, mais seulement si l'on est prêt à y mettre toutes ses forces, chaque muscle, la moindre énergie. [...] Approcher mes mains et mon nez du béton était la seule façon de comprendre sur quoi reposait le pouvoir, le vrai ${ }^{51}$.

Il raconte comment les ouvriers laissent agoniser et mourir un collègue après un accident de travail de peur que l'arrivée d'une ambulance n'enclenche une inspection judiciaire du chantier illégal. Chaque pas dans la ville devient alors un rappel physique à l'ordre économique impitoyable: "J'essaie toujours de calmer cette anxiété qui s'empare de moi quand je marche, chaque fois que je monte un escalier, que je prends un ascenseur, que j'essuie mes chaussures sur un paillasson et que je franchis le seuil d'une porte. Je ne peux m'empêcher de ressasser éternellement la façon dont ont été construits maisons et immeubles. ${ }^{52}$ Le pouvoir de la camorra et la difficulté de représenter celui-ci sont symbolisés par le béton: une matière omniprésente, une base sans laquelle la ville s'effondrerait, et pourtant elle est capable de se faire discrète, d'être partout sans se faire remarquer. Elle épouse toutes les formes et toutes les couleurs, elle n'a ni goût ni odeur. Pour Saviano, cette pierre fluide est la marque sensuelle de sa région :

Peut-être a-t-on un rapport singulier, unique, avec certaines substances, lorsqu'on est né sous certaines latitudes. La même matière est vue de façon différente dans chaque lieu. J'imagine qu'au Qatar l'odeur du pétrole et de l'essence stimule des sensations et des saveurs qui évoquent des villas luxueuses, des limousines et des lunettes de soleil. [...] Dans le sud de l'Italie, c'est la même chose pour le ciment. Le pétrole du Sud. Tout vient du ciment ${ }^{53}$.

La neutralité du béton sur le plan sensuel lui permet de renfermer des substances dangereuses: Saviano raconte comment un chef de chantier avait

\footnotetext{
${ }^{51}$ Saviano, Gomorra, 324.

${ }^{52}$ Ibidem, 328.

${ }^{53}$ Ibidem, 329.
} 
pendant dix ans pour tâche de repartir une poudre toxique dans le ciment. Les déchets sont bétonnés dans les murs où ils répandent, doucement mais sûrement, leur poison. Les sens, dont une fonction évolutionnaire était d'avertir le corps contre le danger, sont court-circuités d'une manière guère imaginable pour les générations antérieures. Saviano dit "sentir» le crime organisé dans chaque grain de béton, mais en réalité, c'est une question de savoir. «Et donc, quand je monte des marches, quand je traverse une pièce, je n'arrive pas à ne pas sentir. Parce que je sais. C'est une perversion. $»^{54}$

Saviano présente la plus flagrante perversion du rapport entre l'homme et son environnement dans le dernier chapitre de son livre. Il en vient à expliquer comment l'industrie des déchets est devenue une des sources de revenu les plus importantes de la camorra depuis les années 1990. Aujourd'hui, la Campanie est parsemée de décharges clandestines souvent toxiques, stations terminales d'un système économique qui sépare rigoureusement les lieux de production, de consommation et de décomposition.

Saviano emploie à nouveau des métaphores charnelles pour situer la problématique dans le monde sensible :

Les déchets avaient fait enfler le ventre du sud de l'Italie. Un ventre gravide qui s'arrondissait mais dont rien ne sortait jamais, sinon de l'argent, avant d'être de nouveau gravide et d'accoucher d'autres sommes colossales, et ainsi de suite, jusqu'à ce que le corps se dégrade, que les artères se bouchent, que les bronches soient obturées et les synapses détruites, encore et toujours ${ }^{55}$.

Son ultime effort pour se représenter le cours de l'économie est de se rendre en plein milieu des déchets, quitte à se faire intoxiquer par la fumée noire qui marque les décharges où la camorra paie cinquante euros par tas brûlé à des garçons tziganes qui y mettent le feu. La visite de cette "terre des feux » est racontée par l'auteur comme un corps-à-corps avec l'ennemi surpuissant, tellement puissant qu'il a refaçonné le paysage, qu'il a créé de nouvelles matières, couleurs et odeurs :

Un jour, j'avais décidé de traverser à pied la terre des feux. Je m'étais couvert la bouche et le nez d'un mouchoir, comme le faisaient les jeunes Tziganes qui mettent le feu aux tas d'ordures. [...] Je marchais sur un sol rongé par la dioxine, dont les trous étaient remplis par les camions puis libérés par les flammes, pour qu'ils ne soient jamais comblés. [...]La fumée que je traversais n'était pas dense, c'était comme une

\footnotetext{
${ }^{54}$ Saviano, Gomorra, 336.

${ }^{55}$ Ibidem, 428.
} 
substance collante qui se déposait sur la peau et procurait une sensation d'humidités6.

Il commence à pleuvoir et le sol se transforme en un marécage où Saviano s'enfonce jusqu'aux cuisses :

J'étais complètement trempé mais toute la pluie qui coulait sur moi ne parvenait pas à éteindre la brûlure montant de mon estomac et irradiant jusqu'à la nuque. Je voulais savoir si les sentiments humains pouvaient affronter une machine aussi puissante, s'il existait un quelconque moyen d'action, s'il y avait une solution pour échapper aux affaires, pour vivre en dehors des dynamiques de pouvoir. Je me torturais, j'essayais de comprendre si l'on avait une chance de comprendre, de découvrir, de savoir, sans être dévoré et broyé.

La dramaturgie du texte culmine dans cette scène finale. Saviano fait une sorte de profession de foi contre la camorra : «Je suis né en terre de camorra », dit-il, une terre « [o]ù tout a la saveur de l'ultime bataille » et où combattre les clans serait incomparable aux autres luttes d'émancipation, «quelque chose de plus essentiel, de plus viscéral, de charnel ». La mise en jeu du corps propre est donc postulée comme une condition pour entrer dans la bataille. Si l'on ne se met pas en danger tout entier, on ne pourra jamais entièrement se représenter les forces de l'ennemi et encore moins le combattre. Depuis la publication de Gomorra, Saviano vit sous la protection de l'état italien. L'image qui conclut son roman le montre agrippé sur un réfrigérateur flottant dans les ordures. Il hurle : «Maladetti bastardi, sono ancora vivo !» 57.

\section{CONCLUSION : NAPLES AUX NAPOLITAINS}

Les écrivains qui ont fait l'objet de cet article ont visité ou vécu à Naples dans des contextes et avec des intérêts très différents. Le point de comparaison pertinent consiste dans l'expérience sensible de l'espace urbain que chacun d'eux a inscrit dans ses textes. Dans son ensemble, le corpus révèle des changements importants qui concernent les concepts de "ville» et d' « expérience ». Comment le paysage urbain sensoriel a-t-il évolué à travers le temps ?

Avoir un corps ou être un corps dans la ville n'a pas la même signification en 1778 et en 2008 : la place des sensations a évolué, les explorateurs ne se contentent plus d'observer, ils veulent sentir avec leur cinq sens, ou même risquer leur corps entier pour être aux prises avec ce qui définit la vie d'une ville. Une ville, ce n'est plus seulement un patrimoine architectural et artisti-

\footnotetext{
${ }^{56}$ Ibidem, 453.

${ }^{57}$ « Maudits bâtards, je suis encore vivant!».
} 
que, ce sont les usages sociaux, ce sont les goûts et les odeurs de ses rues, ce sont les pratiques artisanales et industrielles sensibles et les conditions socioéconomiques qui agissent en discrétion, soustraites à l'expérience du corps.

Nous savons plus sur les sens aujourd'hui, nous sommes devenus plus exigeants, mais aussi plus prudents : nous voulons sentir plus et nous protéger davantage. Ainsi s'explique une demande accrue de formes d'expérience à la fois plus aventureuses et plus sécurisées. Les autochtones d'une ville sont souvent négligés dans cette équation alors que ce sont eux et leurs pratiques qui donnent du goût à l'expérience urbaine. Si une ville est la somme des sensations qu'elle procure, toute politique urbaine qui appauvrit directement ou indirectement cette sensualité est une politique ratée. La politique patrimoniale devrait en premier lieu prendre acte de la dimension sensuelle de l'endroit qu'elle entend promouvoir ou protéger. Et pourquoi ne pas se tourner vers la littérature à cette fin? Nous avons vu qu'elle peut exceller dans la représentation de toutes les strates de la sensualité locale. Lire un poète comme Malaparte nous ouvre les yeux, les oreilles et les narines sur ce que c'est que sentir une ville. Lire Saviano nous démontre que la surface sensible de nos villes est devenue tellement lisse que nous ne sentons plus les forces qui gèrent le fond. Avoir un corps dans la ville aujourd'hui signifie d'abord être conscient de la dialectique de sentir et savoir, chercher la sensation pour compléter et renforcer la connaissance.

Concrètement, pour le cas de Naples, ceci veut dire qu'une future politique urbaine devra s'engager dans la lutte anti-mafia pour libérer la population et protéger l'environnement sans pour autant céder à la solution d'une ville purgée à l'instar de Barcelone ou de Venise où les sensations seraient successivement normalisées. La qualité de vie des autochtones et la qualité d'expérience des touristes seraient détruites si le changement de Naples s'effectuait par une normalisation sensuelle de la ville. Rendre une ville accessible, l'assainir, l'ouvrir à l'expérience de tous n'est pas en soi un bon ni un mauvais projet. Il importe de le mener dans le respect des usages déjà présents. Là où la ville s'aseptise, s'insonorise, exclut les odeurs et les standardise les saveurs, elle perd son goût, elle détruit son histoire, elle renonce au partage du sensible entre habitants et visiteurs. Elle devient la proie d'une nouvelle pollution et de nouveaux abus de pouvoir.

Le grand défi, c'est de libérer Naples de la camorra en conservant sa diversité urbaine qui est une diversité sensuelle. Rendre Naples aux napolitains, pour que des poètes moins tristes que Saviano puissent le chanter bientôt. 